\title{
AN EXPLORATORY STUDY ON CHARACTERS AND RELATIONSHIPS AMONG PARTICIPATION MOTIVATIONS AND RELATED ELEMENTS OF YOUTH BASKETBALL PLAYERS
}

\author{
Howard Z. Zengi \\ Dr. and Professor, \\ Department of Kinesiology, \\ Brooklyn College of The City University of New York,
} USA

\begin{abstract}
:
Problem Statement: Since the Youth Olympic Summer Games became a new global sports event in 2010 and is held every four years, youth athletes' behaviors and factors that motivated them to continually participate in the sport they choose have become interesting research inquiries: what are the reasons/factors that really motivated them continually engaged in the sports they love? Can these reasons/factors be identified and predicted? Purpose: The purposes of this study were to examine the traits of essential factors that motivated the youth basketball players to participate in their practices and competitions; examine how Gender, Supporting, Goal-setting, Years Playing Basketball related elements impacted their levels of participation-motivations; and the relationships among these psychological needs and motivation factors. Furthermore, the predictors among the psychological needs associated with their essential motivation factors were also explored. Methods: The Adapted Youth Basketball Players' ParticipationMotivations Questionnaire was used for data collection; and Self-Determination Theory was used as the theoretical framework; the participants were 253 youth basketball players (Boys $=136$, Girls = 117). Data analyses were done by a $2 \times 2 \times 2 \times 2$ factorial MANOVA, exploratory factor analysis, and multiple regression analyses. Results: Findings indicate that Supporting and Goal-setting elements had significant effects on the youth basketball players' participation-motivations, but Gender and Years Play Basketball elements did not. The youth basketball players who were supported by parents possessed higher participant motivations than those supported by their schools. The participants who set their goals for professionals possessed higher participation-motivations than those who set goals for non-professional. Conclusions: In conclusion, through multiple statistics approaches and analyses, the traits and relationships among the youth basketball players' participation-motivations, and how their motivations associate with the four related elements can be identified and predicted.
\end{abstract}

i Correspondence: email hzeng@brooklyn.cuny.edu 
Keywords: self-determination; goal-setting; coaching; management

\section{Introduction}

Dr. James Naismith invented the basketball game since January 20th, 1892 in Springfield, Massachusetts, US. This sport has become one of the most popular, enjoyable, and highest economic income-producing team sport on earth. This is why a well-known sportswriter described that: From New York to London, from Paris to Beijing, from Sarajevo to Buenos, and from Aires to Los Angeles ... The game is played wherever someone can get a ball, and amounted ring-like object; and also played in those expensively designed courts of the NBA. Unlike other sports that require multiple people and a lot of equipment, the game of Basketball only requires a ball, a hoop, and the motivation to play (Faurschou, 2015; Basketball Player Positions, 2017). When exploring what sports are played in schools and physical education programs, you will find basketball on their curricula list. From grade three to grade twelve to college or university, basketball skills, tactics, or strategies are taught and learned on different levels of proficiency (Geidne et al., 2013). This phenomenon clearly reflects that the sport of basketball deeply involves areas of education, entertainment, and the economy.

\subsection{Youth Olympic Games}

The Youth Olympic games (YOG) became a new global sports event in 2010, and is held every four years; this sports event has added a new hope for those teenagers who have a sport's dream (Official Report, 2010). For the teenagers, who can compete in the YOG, win the recognition to enter a higher competitive sports arena or obtain a scholarship to play on the college/university level, which means they will have a bright future. This might be one of the reasons why research demands on youth athletes' participation motivations are getting more important and urgent. An exciting fact in the USA is that: after being selected as the USA youth Basketball team members competed in the YOG, those youth basketball players (YBPs) will have a very high chance be enrolled in a college or university, and to play in a National Collegiate Athletic Association (NCAA) division team (NCAA, 2018; NYCYBL, 2018; NYCDE, 2018). In other words, these Youth basketball players will start his/her collegiate athlete journey. All of these were the reasons why research studies about YBPs have become increasingly broader and deeper (NYCYBL, 2018) in recent years.

\subsection{Why Conducting This Study}

The research literature in youth sports has attributed the goals and reasons why youth athletes participate in their practices and competitions to enjoyment, physical health, having fun, foster self-esteem, friendship, passion or love the game, and peer acceptance, to contest winners, to become a coach, to satisfy family's will, etc. (e.g., Breese, 1998; Claver et at. 2017; Geidne, Quennerstedt, \& Eriksson, 2013; Gonzalez-Cutre \& Sicilia, 2012; Lippitt, 2012; Miguel \& Machar, 2007; McPherson, 2015; Zeng, 2018a; Zeng et al., 
2019). The review of research literature from the youth sport domain, however, lacks solid evidence about what motivation factors or reasons have really motivated YBPs who continually participate in their practices and competitions. Therefore, this study was aimed at collecting such evidence so that the professionals (i.e., coaches, teachers, and managers) have much clearer information, knowledge, and ideas for youth basketball teams/clubs.

\section{Literature Review}

\subsection{Self-Determination Theory}

The theoretical framework that guided this study was the Self-Determination Theory (SDT, Ryan \& Deci, 2000). Based on the motivation origin sources, SDT established two different motivation types, intrinsic motivation, and extrinsic motivation; this classification allows for a greater understanding of why peoples want to take part in and continually engage in an activity (exercise or sport) (Gonzalez-Cutre \& Sicilia, 2012). Researchers further indicated that all people possess multiple motivations, both intrinsic and extrinsic; these two motivations must simultaneously in play and work together to be determined and accomplished the overall quality of motivation (Gonzalez-Cutre \& Sicilia, 2012; Stellion \& Sinclair, 2013) [9]. Hence, athletes actually were motivated by three psychological needs: competence, relatedness, and autonomy (Gonzalez-Cutre \& Sicilia, 2012; 17-Stellion \& Sinclair, 2013).

In the SDT model, the Competence Needs is called effectiveness motivation; while the Relatedness Need refers to people's needs, and belong to feel accepted by others; however, the Autonomy Needs to refer to people's need to feel Self-Determined that is the source of persons' own action (Ryan \& Deci, 2000; Stellion \& Sinclair, 2013). The Organismic Needs energize intrinsic and extrinsic motivations. Researchers, however, indicated that the concept of need is too general and vague to illustrate the participation in particular behaviors and it is hard to guide empirical research (Kaplan, 2010; Pintrich \& Schunk, 2002). Therefore, a few models describing how different motivations triggered by need manifest in intrinsic and extrinsic motivation in specific aspects or activities were developed (Stellion \& Sinclair, 2013; Kaplan, 2010; Pintrich \& Schunk, 2002).

Breese (1998) and Cox (2011) stated that athletes' Intrinsic Motivations could be defined as participating in a sport for fun or enjoyment, while their Extrinsic Motivation could be defined as they were participating in a sport to gain rewards. Further, when athletes begin to engage in a particular sport, they are motivated not only by Intrinsic Motivations but also by Extrinsic Motivations (Breese, 1998; Cox, 2011). For some particular sports, however, may be dependent on more Intrinsic Motivations than those of Extrinsic Motivations (Breese, 1998; Cox, 2011); about this viewpoint, the following researchers have a similar vision on this regard. The reasons were different types of sports need different types of motivations (Duricek \& Cynarski, 2017; Gonzalez-Cutre \& Sicilia, 2012; and Stellion \& Sinclair, 2013). 
More specifically, other researchers illustrated that athletes' Intrinsic Motivations usually predict athletes' attendance and adherence to a particular sport (Chen et al., 2014; Smith et al., 2006). Additionally, the research literature is quite consistent with respect to the benefits of Intrinsic Motivations to learning and development; that is, engagement based on Intrinsic Motivations does not need external incentives or rewarding and was able to enhance the motivations necessary to engage in the same activity again and again in the future (Smith et al., 2006; Zeng, 2018). In this study, youth athletes who are intrinsically motivated would be those who go to practice their techniques and fitness regularly for fun and for self-satisfaction; whereas those youth athletes who are extrinsically motivated would be those who go to practice to become better YBPs for winning a medal in the competition (Kaplan, 2010; Zeng et al., 2019). It is interesting to know that Intrinsic and Extrinsic Motivations have different effects on YBPs (Stellion \& Sinclair, 2013; Zeng et al., 2019). In the present study, the researcher is trying to find the evidence and factors that motivated the YBPs' to participate in the sport of basketball.

Also, this study wanted to explore how educators (coaches, teachers, program managers) apply the SDT to enhance their coaching, teaching, and administration. Kaplan (2010) in his review of Intrinsic and Extrinsic Motivation summarized: now we know the variation exists is important, and it has been a widespread consensus among researchers and educators that enhancing intrinsic motivations among athletes or students is beneficial (Kaplan, 2010). Youth players' intrinsic motivations will be enhanced when practices promote their sense of personal autonomy, when teamwork is challenging and relevant to all team members when social relationships are supportive, and when the environments are physically and psychologically safe. Kaplan (2010) and Lippitt (2012) further illustrated, how practices that promote these environmental characteristics include providing athletes with choices among activities and between ways of completing tasks, encouraging athletes to explore and pursue their ambition, based on their backgrounds and prior experiences developing their tasks, encouraging them to collaborate, incorporating fantasy in activities, providing feedback that is informative and frequent, and reducing external rewards.

In the real training process, however, youth athletes or players are required to participate in tasks that they are not motivated to do or do not understand why they have to do. Under these situations, Extrinsic Motivation should be applied for motivating them to execute those tasks (Kaplan, 2010; Lippitt, 2012; Zeng, 2018 ). However, physical educators should pursue the internalization of their Intrinsic Motivations for these tasks as well. Such internalization can be promoted by employing many necessary for illustrations to the player prior to having them executing those tasks (Kaplan, 2010; Lippitt, 2012; Ryan et al., 1997; Zeng, 2018a). Additionally, coaches or teachers should make the values of these tasks explicit and clearer. These can be done most effectively through modeling and by providing a clear and age-appropriate rationale for youth athletes (Kaplan, 2010; Lippitt, 2012; Stellion \& Sinclair, 2013; Ryan et al., 1997; Zeng, 2019). 


\subsection{Research Purpose and Questions}

To further clarify and understand the issues described above, the purposes of the present study were: a) examining the Characters of essential factors that motivated the YBPs participating in their practices and competitions; b) examining the relationship between the YBPs' 'three psychological needs' associated with their Gender, Supports, Goalsetting, Years Play Basketball elements; and c) exploring the predictors relate to their psychological needs, motivation factors, and those related elements. The following research questions guided the present study: (1) what motivation factors motivated the YBPs to participate in basketball practices and competition? (2) What would be the Characters and correlation among the 19 motivation factors variables? (3) Do differences exist in the motivation scores between who supported by parents or by the school? (4) Do differences exist in the motivation scores between the participants' Goal-Settings (for professional or non-professional)? (5) Do differences exist in the motivation scores between the participants' gender? (6) Can the participants' three psychological needs or their essential motivation factors be predicted using multiple regression analysis techniques?

\section{Materials and Methods}

\subsection{Participants}

The participants were selected from 12 high schools in New York City (NYC), before recruiting the participants, the approval for using human subjects in this study was granted by the Institutional Review Board (IRB). Students' enrollment of these selected 12 high schools were from 1,089 to 2,675; all these schools are under the administration of the NYC Department of Education. These schools agreed and shared the following statement for their basketball teams.

As a youth basketball league, we take great pride in sending our young men onto colleges or universities and even in some cases to the NBA. Since our leagues were established, we have been implementing the following educational idea: More importantly, though it is our heightened sense of responsibility in teaching children the values of hard work, dedication, and the importance of education. We strongly believed that: Today's your peoples will become future leaders and we are proud to be a part of that mentoring process by the high school basketball leagues in this city (NYCYBL, 2018).

\subsection{Procedure}

The procedure of this study included: 1) obtained approval for conducting this survey study from the Institutional Review Board (IRB) of the College; 2) under the coach's supervision, the researcher administered the questionnaires, explained how to completed it, and resolved all questions that arose during the survey process; 3) an envelope for preventing others and coach from viewing the answers on the questionnaire was provided; 4) the participants signed the Informed Consent Form and returned it to the researcher. All coaches were informed that after completing this study, the outcomes 
would be shared with them. As a result, among the 300 questionnaires delivered, 253 were correctly completed and returned to the researcher (return rate $=84.3 \%$ ).

\subsection{Data Collection and Analysis}

The Adapt Youth Basketball Players' Participation Motivations Questionnaire (AYBPPMQ, Zeng, 2019) was employed for data collection. This questionnaire contains two parts: Part I asked General Information. It contains eight questions that questionnaire covered participant's general information, such as: How long have you officially play basketball? Financially, who supported you as you engaged in basketball practices and competitions? Part II asked: What factors/reasons motivated you to take part in basketball practices and competitions continually? With 19 motivation factors provided, the participant can respond to each motivation factor (MF) on a 5-point Likert type scale (5points represents Strongly agree, and 1-point represents Disagree). Part II of the questionnaire contains 10 Intrinsic Motivations factors (items 1, 2, 4, 7, 8, 10, 13, 14, 15, and 17) and nine Extrinsic Motivations factors (items 3, 5, 6, 9, 11, 12,16, 18, and 19). In other words, it included all three basic psychological needs (Competence, Relatedness, and Autonomy) described by Ryan and Deci (2000). According to Child's (1990) suggestion, to explore the possible underlying factors of the structure for a set of measured variables without imposing any preconceived structure on the outcome, the exploratory factor analysis (EFA) is an ideal option. Hence, this study employed the EFA and implemented this method to determine what independent variables would be included in the questionnaire and enter the multiple regression analysis (Chen et al., 2014; Child, 1990). The results revealed the analysis extracted six factors with very well correspondence to the 19 items with eigenvalues for the reasons or factors ranging from 2.67 to 8.61 and structure coefficients from .77 to .91 and the majority of the fitted residuals reached the pre-set-up significant difference $(P<.05)$ level (Child, 1990). Additionally, the validation process was through a pilot study, reviewing the content or items. These processes confirmed the following concerns: a) the reading and writing ability of the youth basketball players (15-18 years old); b) whether or not those youth basketball players can truly understand and respond to the questions in the questionnaire correctly; c) it may result in re-wording on some questions or statements to improve the understanding for the participants or cutting or adding numbers of the questions or statements in the questionnaire; and d) whether or not the questions covered all the possible motivating factors for the youth basketball players who participated in basketball practices and competitions.

In terms of date analyses, first, descriptive statistics were conducted for reflecting the general status of how the participants were motivated to participate in basketball practices and competitions. Second, to determine the status of correlation among the 19 MFs variables, correlation analysis among all variables was conducted. Third, to look at the effects of four independent variables Gender (boys, girls) x Supports (by parents, by school) x Goal-settings (for professional, for non-professional) x Years play Basketball (23 years, 4 \& more years) on the 19 independent variables, the $2 \times 2 \times 2 \times 2$ factorial 
MANOVA was performed. Last, to examine the relationships between the three psychological needs satisfaction for the motivation factors; the Explore factor analysis (EFA) and the multiple regression analyses were employed to assist in predicting the three psychological needs and the motivation factors scores of the youth basketball players. The statistical program utilized was the IBM Statistics Package for the Social Science Version 25.

\section{Results and Discussion}

Based on the research questions of this study: 1) what factors motivated the YBPs to participate in the sport of basketball? 2) What kind of correlation status existed among the 19 motivation factors? 3) If differences exist in the motivation scores among the participants' Supports, Goal-setting, Gender, and Years play Basketball? 4) If the participants' three psychological needs could be predicted using the multiple regression analysis approaches? The findings are presented in the Table 1.

\subsection{Participants' General Information}

Table 1 reflects "Participants' General Information". The participants reported they have played basketball for at least three years $(131 / 51.78 \%)$, and four or more years $(122 / 48.22 \%)$. The mean BMI for boys was $22.98( \pm 9.23)$ and for girls, it was $21.67( \pm 5.76)$. Note that players from the high school basketball team represent the highest skill and competitive capability in the youth competitive sport system of the United States. For example, many of the players drafted into the NBA were high school students, including NBA legends - LeBron James and Carmelo Anthony - both of them are now regarded as the most successful players in NBA history. Before they were drafted into the NBA, their success stories and recorded have attracted thousands of youth basketball players (NBA Draft, 2016). The high school basketball teams practice at least five half days per week, depending on the seasons, times of practice for each day varied. If necessary (determined by coaches), they may have a morning exercise in which most likely is focus on fitness of body conditioning.

Table 1: Participants' General Information $(\mathrm{N}=253$, Boys $=136$, Girls $=117)$

\begin{tabular}{|l|l|}
\hline Number / Questions & \multicolumn{1}{|c|}{ Answers / Frequency / Percentage } \\
\hline 1. What is your gender? & $\begin{array}{l}\text { Boys }=136 / 53.75 \% \\
\text { Girls }=117 / 46.25 \%\end{array}$ \\
\hline $\begin{array}{l}\text { 2. What grade levels are you } \\
\text { currently going to? }\end{array}$ & $\begin{array}{l}\text { Grades } 9-10=102 / 40.32 \% \\
\text { Grades } 11-12=151 / 59.68 \%\end{array}$ \\
\hline $\begin{array}{l}\text { 3. How long have you officially } \\
\text { play basketball? }\end{array}$ & $\begin{array}{l}\text { One year }=0 / 0 \% \\
\text { Two years }=0 / 0 \% \\
\text { Three year }=131 / 51.78 \% \\
\text { Four or more years }=122 / 48.22 \%\end{array}$ \\
\hline 4. What is your BMI? Note & $\begin{array}{l}\text { Answer for boys: Mean BMI }=22.98( \pm 1.53) \\
\text { Answer for girls: Mean BMI }=21.67( \pm 1.71)\end{array}$ \\
\hline 5. What is your age-range? & $15-16=119 / 47.04 \% 17-18=134 / 52.96 \%$ \\
\hline
\end{tabular}




\begin{tabular}{|l|l|}
\hline \hline $\begin{array}{l}\text { 6. Financially, who supported you engaged } \\
\text { in basketball practices and competitions? }\end{array}$ & $\begin{array}{l}\text { By my parents }=136 / 53.75 \% \\
\text { By my school/team }=117 / 46.25 \%\end{array}$ \\
\hline $\begin{array}{l}\text { 7. What is your goal of engaging in } \\
\text { basketball practices and competitions? }\end{array}$ & $\begin{array}{l}\text { For become a professional basketball player }=88 / 34.78 \% \\
\text { For a non-professional basketball player }=165 / 65.22 \%\end{array}$ \\
\hline
\end{tabular}

Note. Using the formula of BMI $=\mathrm{kg} / \mathrm{m}^{2}$

\subsection{Descriptive Statistics}

The mean scores and standard deviation for all 19 motivation variables in participants' motivation scores are presented in Table 2: As showed in Table 2, the top six factors were motivation factor (MF) MF1 Unique content \& value, MF17 Unique sport skill, MF3 For healthier, MF4 Enjoyment and happiness, MF12 Reputation, and MF9 Improve physical health. The grand mean $\left(\mathrm{MG}_{\mathrm{G}}\right)$ of these six MFs is $\mathrm{MG}_{\mathrm{G}}=4.005$ and possessed the highest impact power on these youth basketball players' (YBPs) participation motivation. The bottom seven factors were MF15 Reduce pressure, MF5 Meet my friends, MF13 Establish prestige, MF14 Get the recognition, MF10 For professional, MF18 Become a coach, and MF19 Satisfy family's will. The grand mean of these seven factors is $M_{G}=2.980$ and these seven factors possessed lowest or less impact power on these YBPs' motivation. The mean score of other six factors was at the medium level. These MFs were: MF2 Having fun, MF6 Make new friends, MF8 Shape body, MF16 Reduce troubles, MF7 Contest winners, and MF11 Foster self-esteem. The grand mean of these six factors is $M_{G}=3.526$ and these six factors possess medium impact power on these YBPs' participation motivations (See Table 2).

Table 2: Means and Standard Deviations of all Variables $(\mathrm{N}=253$, Boys $=136$, Girls $=117)$

\begin{tabular}{|l|c|c|c|}
\hline Motivation Factors (MF) & Mean \pm S.D. & Sum & Rank \\
\hline MF 1 Because basketball's high technical content and unique value. & $4.502 \pm .774$ & 1139.006 & 1 \\
\hline MF 2 For the fun and get rid of boredom. & $3.660 \pm 1.313$ & 925.980 & 7 \\
\hline MF 3 For getting healthier whole body & $4.114 \pm .867$ & 1014.884 & 3 \\
\hline MF 4 For the enjoyment and have happiness. & $3.897 \pm .974$ & 985.941 & 4 \\
\hline MF 5 In order to meet my friends. & $3.375 \pm 1.111$ & 853.875 & 14 \\
\hline MF 6 In order to make new friends. & $3.644 \pm .975$ & 921.932 & 8 \\
\hline MF 7 In order to contest winners. & $3.510 \pm 1.167$ & 888.030 & 11 \\
\hline MF 8 In order to shape the body. & $3.596 \pm .948$ & 909.788 & 9 \\
\hline MF 9 In order to improve physical health & $3.668 \pm 1.039$ & 928.004 & 6 \\
\hline MF 10 For become a professional player. & $2.672 \pm 1.148$ & 676.016 & 17 \\
\hline MF 11 In order to foster self-esteem. & $3.415 \pm 1.125$ & 863.995 & 12 \\
\hline MF 12 In order to improve my own reputation & $3.703 \pm 1.051$ & 936.859 & 5 \\
\hline MF 13 In order to establish prestige among my friends. & $3.296 \pm 1.099$ & 833.888 & 15 \\
\hline MF 14 In order to get the recognition from my teacher/coach. & $3.241 \pm 1.095$ & 819.973 & 16 \\
\hline MF 15 In order to reduce the learning pressure. & $3.379 \pm .975$ & 854.887 & 13 \\
\hline MF 16 To reduce the troubles from learning/work. & $3.545 \pm .910$ & 896.885 & 10 \\
\hline MF 17 In order to develop a unique sport skill. & $4.146 \pm .975$ & 1048.938 & 2 \\
\hline MF 18 Hope to become a basketball coach in future. & $2.589 \pm 1.415$ & 655.017 & 18 \\
\hline MF 19 In order to satisfy the will of family. & $2.308 \pm 1.123$ & 583.924 & 19 \\
\hline
\end{tabular}

Note: a) S.D. = Standard Deviation. b) The motivation factor (MF) 1, 2, 4, 7, 8, 10, 13, 14, 15, and 17 are 'Intrinsic motivation factors'. c) The MF 3, 5, 6, 9, 11, 12, 16, 18, and 19 are 'Extrinsic motivation factors'. 


\subsection{Differences among the Related Elements}

The interpretation of the results of $2 \times 2 \times 2 \times 2$ factorial MANOVA for the YBPs' MFs released that: no significant difference in the Gender element $(p>.05), \Lambda=.906, F=1.240$; and no significant difference in the Years play Basketball element $(p>.05), \Lambda=.901, F=$ 1.172 either; however, a significant differences effect was found in Supports and Goalsetting elements with $(p<.011), \Lambda=.895, F=1.959$ and $(p<.000), \Lambda=.769, F=3.594$ respectively. According to the research design, after significant differences effects were found, a following up MANOVA test was executed. This following up test determined what variables or motivation factors that truly motivated these YBPs engaged in their practices and competition (See Table 3).

Table 3: Comparison of YBPs' Motivation Scores after the 2 × 2 MANOVA

Significant Difference Effects Revealed in 'Supports' and 'Goal-Setting' $(\mathrm{N}=253)^{\text {Note }}$

\begin{tabular}{|c|c|c|}
\hline MF & $\begin{array}{c}\text { Supports-by Mean } \pm S D \\
\text { Parents }(n=136) \text { vs. School }(n=117)\end{array}$ & $\begin{array}{l}\text { Goal-Settings Mean } \pm S D \\
\text { Professional }(n=88) \text { vs. N-Professional }(n=165)\end{array}$ \\
\hline MF1. & $4.713 \pm .5694 .256 \pm .901^{*}$ & $4.807(.499) 4.339 \pm .844^{*}$ \\
\hline MF2. & $3.647 \pm 1.4273 .675 \pm 1.173$ & $3.432 \pm 1.3323 .782 \pm 1.153$ \\
\hline MF3. & $4.398 \pm .7523 .773 \pm .886$ & $4.329 \pm .8544 .000 \pm .883$ \\
\hline MF4. & $4.037 \pm .9613 .735 \pm .968$ & $4.079 \pm .9613 .800 \pm .970$ \\
\hline MF5. & $.566 \pm 1.1393 .153 \pm 1.039 *$ & $3.773 \pm 1.0903 .163 \pm 1.066^{*}$ \\
\hline MF6. & $3.713 \pm .9653 .654 \pm .986$ & $3.897 \pm .8583 .509 \pm 1.009$ \\
\hline MF7. & $3.654 \pm 1.1183 .342 \pm 1.204$ & $4.057 \pm .9013 .218 \pm 1.189^{*}$ \\
\hline MF8. & $3.735 \pm .8883 .436 \pm .994$ & $3.772 \pm .7693 .503 \pm 1.021$ \\
\hline MF9. & $3.779 \pm 1.0723 .538 \pm .987$ & $3.670 \pm .9673 .667 \pm 1.078$ \\
\hline MF10. & $3.132 \pm 1.1342 .136 \pm 1.332^{*}$ & $3.932 \pm 1.1912 .000 \pm 1.263^{* *}$ \\
\hline MF11. & $3.617 \pm 1.1923 .179 \pm .996^{*}$ & $3.909 \pm 1.1613 .152 \pm 1.015^{*}$ \\
\hline MF12. & $3.823 \pm 1.0743 .564 \pm 1.011$ & $4.057 \pm .9513 .515 \pm 1.056^{*}$ \\
\hline MF13. & $3.382 \pm 1.1673 .197 \pm 1.010$ & $3.636 \pm 1.0413 .115 \pm 1.090^{*}$ \\
\hline MF14. & $3.338 \pm 1.1623 .128 \pm 1.004$ & $3.534 \pm 1.0493 .084 \pm 1.089^{*}$ \\
\hline MF15. & $3.507 \pm .9583 .231 \pm .977$ & $3.432 \pm .8553 .351 \pm 1.034$ \\
\hline MF16. & $3.632 \pm .9173 .444 \pm .894$ & $3.591 \pm .8923 .521 \pm .921$ \\
\hline MF17. & $4.389 \pm .7813 .863 \pm 1.097^{*}$ & $4.421 \pm .7724 .000 \pm 1.059^{*}$ \\
\hline MF18. & $2.985 \pm 1.4652 .128 \pm 1.201^{*}$ & $3.443 \pm 1.3632 .133 \pm 1.222^{* *}$ \\
\hline MF19. & $2.434 \pm 1.1842 .162 \pm 1.033$ & $2.488 \pm 1.0932 .212 \pm 1.123$ \\
\hline
\end{tabular}

Note: The above results are from the follow-up test: a) Gender and Years play Basketball element did not reach a significant difference at the $p<.05$, hence, these two elements' results did not show here. b) In Supporting element, six out of 19 comparisons reached significant differences at $p<.05^{*}$ Level. c) In the GoalSetting element, ten out of 19 comparisons reached significant differences wherein eight comparisons reached significantly different at $p<.05^{*}$ level and two at significant different $p<.01^{* *}$ Level. d) $\mathrm{MF}=$ Motivations Factors.

\subsection{Internal Consistencies and Correlation Analyses}

First, in the study of "Sadly, the earth is still round ( $p<0.05)$ " (Zhu (2012), the author indicated that the criteria of correlations degree for low correlation is $r=.20-.39$; for moderate correlation is $r=.40-.59$; for moderately high correlation is $r=.61-.79$; and for high correlation is $r \geq .80$. e) There are total of 71 MFs correlation coefficients reached 
significant at $\mathrm{p}=.05^{*}$, and $\mathrm{p}=.01^{* *}$ level. Moreover, according to the criteria recommended above by Dr. Zhu (2012), the current study performed the EFA and MRA; the results revealed that from the entire 171 correlation coefficients examined, there was a total of 71 correlation coefficients reached significant levels (at $p=.05^{*}$ and $p=.01^{* *}$ levels separately). Furthermore, the EFA determined 15 variables could be accepted to enter the regression analyses.

\subsection{The Predictors of Three Psychological Needs}

According to the study findings from two previous research groups (Chen et al., 2014; Stelluno \& Sinclair, 2013), students' motivations to participate in after school physical activities or sports could be effectively predicted through using the techniques of exploratory factor analysis (EFA) and multiple regression analyses (MRA); appreciated and accepted their recommendations, the present study also applied these two techniques (see Table 4 for details).

Table 4: Unstandardized Regression Coefficients (Beta in parentheses) for the participants' Motivation Factors (MF) Standardized Score $(\mathrm{N}=253)$

\begin{tabular}{|c|c|c|c|}
\hline Predictor Variables Note & $\begin{array}{l}\text { DV: Model I } \\
\text { Gender }\end{array}$ & $\begin{array}{c}\text { Model II } \\
\text { Goal-setting }\end{array}$ & $\begin{array}{l}\text { Model III } \\
\text { Supports }\end{array}$ \\
\hline \multicolumn{4}{|l|}{ Competence Needs variable } \\
\hline MF7. In order to contest winners & $044(.102)$ & $-.047(-.145)$ & $023(.054)$ \\
\hline MF8. In order to shape the body & $.086(.164)$ & $.053(.097)$ & $.008(.015)$ \\
\hline MF10. To become professional player & $-.022(-.067)$ & $-.161(-.477)$ & $-.069(-.214)$ \\
\hline MF13. In order to establish prestige & $-.074(-.163)$ & $-.060(-.128)$ & $.015(.034)$ \\
\hline MF18. To become a basketball coach & $.064(.181)$ & $-.013(-.034)$ & $-.036(-.102)$ \\
\hline \multicolumn{4}{|l|}{ Relatedness Needs variable } \\
\hline MF2. Having fun not boredom & $.015(.039)$ & $.024(.061)$ & $.018(.480)$ \\
\hline MF4. For enjoyment \& happiness & $-.047(-.092)$ & $.004(.007)$ & $-.008(-.015)$ \\
\hline MF6. In order to make new friends & $-.044(-.087)$ & $.004(.008)$ & $.015(.029)$ \\
\hline MF12. To improve my reputation & $-.074(-.155)$ & $-.072(-.145)$ & $-.003(-.005)$ \\
\hline MF19. To satisfy the will of family & $.043(.097)$ & $.070(.151)$ & $.011(.025)$ \\
\hline \multicolumn{4}{|l|}{ Autonomy Needs variable } \\
\hline MF3. For healthier whole body & $-.117(-.202)$ & $-.158(-.274)$ & $-.158(-.274)$ \\
\hline MF9. To improve physical health & $.004(.009)$ & $.001(.001)$ & $.001(.001)$ \\
\hline MF11. To foster self-esteem & $.020(.044)$ & $-.029(-.065)$ & $-.029(-.065)$ \\
\hline MF15. To reduce learning pressure & $-.001(-.002)$ & $-.058(-.113)$ & $-.058(-.113)$ \\
\hline MF16. To reduce troubles at school & $.004(.007)$ & $-.001(-.002)$ & $-.001(-.002)$ \\
\hline Constant & $1.988^{*}$ & $2.246^{*}$ & $2.418^{*}$ \\
\hline$\Delta \mathbf{R}^{2}$ & .194 & .314 & .258 \\
\hline $\mathbf{F}$ & $2.753^{*}$ & $8.703^{* *}$ & $4.152^{* *}$ \\
\hline
\end{tabular}

Note: 1) $D V=$ Dependent Variables. 2) According to the results from the exploratory factor analysis, only listed those variables accepted to be entered the regression analyses. 3) $\Delta R 2=$ adjusted R2. 4) The information above is based on the method of listwise deletion of cases. 5 ) ${ }^{*} P=<.05$, and ${ }^{* *}=P<.01$.

In the first set of MRA, the Motivation Factors (MFs) standardized score was the dependent variable; the satisfaction levels of Competence needs, Relatedness needs, and 
Autonomy needs were the independent variables. Results revealed a significant regression, $F=2.753$, at $p<.05$ level; means the combination accounted variance for the three psychological needs satisfaction from the Gender element was $19.4 \%$. In other words, this satisfaction level is just as high as people expect. In contrast, a significant regression appeared from the Goal-setting element, $F=8.703$, at $p<.01$ level; this result demonstrated the combination accounted variance for the three psychological needs satisfaction from the Goal-setting element reached $31.4 \%$; that is, the highest satisfaction in the current study. This finding demonstrated that the Goal-setting at "for professional" or "for non-professional" possesses the highest driven power.

Moreover, the MRA on the Supports element also reached a significant $F=4.152$, at $p<.01$ level; the contribution on the satisfaction to the three psychological needs from this element was $25.8 \%$; this result seems to just like people expected. In summary, all three psychological needs categories reached a significant level (with two at $p<.01$, and one at $p<.05$ level). The total satisfaction percentage was $76.6 \%$ or the "Total Variance Explained" through the EFA and MRA implied that: the physical educators (coaches, instructors, and administrators) who involved in the current study did a pretty good job in cultivating and managing these YBPs. This might be a successful way to encourage and maintain YBPs to continue to participate in their practices and in competitions.

The present study examined the relationships among the 19 participation motivation factors and four related elements of the youth basketball players (YBPs) from NYC, US. The findings revealed: there are differences existed among the participants' motivation scores with regard to their Gender, Years play Basketball, Supporting, and Goal-setting; with Supporting, and Goal-setting two elements reached a significant difference level, but Gender and Years play Basketball did not reach a significant difference level. Relationships between the motivations variables and related elements variables were identified. The results showed that there are positive correlation traits on predicting the satisfaction degrees with regard to participants' three basic psychological needs (including a total of $15 \mathrm{MFs}$, details can be found in Table 5); specifically, higher positive correlation coefficients were found in Goal-setting and Supporting elements.

First, according to data exhibited in Table 2, the MFs scores placements can be divided into three groups: 1) The high impact factors group, containing MF1, MF17, MF3, MF4, MF12, and MF9, possessed the highest score and impact power on this sample's YBPs' motivations. Interestingly, among these six MFs, the MF1, MF4, and MF17 are in the Intrinsic Motivation category, while the MF3, MF12, and MF9 are in the Extrinsic Motivation category. 2) The medium-impact MFs group with medium-high scores, containing MF2, MF6, MF8, MF16, MF7, and MF11, possessed medium impact power on this sample's YBPs' motivations. Similar to the first group, this group also has three MFs (i.e., MFs 2, 8, 7) belong to the Intrinsic Motivation category and three MFs (i.e., MFs 6, 16, 11) belong to the Extrinsic Motivation category. 3) The lower impact MFs group, consisting of MF15, MF5, MF13, MF14, MF10, MF18, and MF19 (with lowest scores), possessed significantly lower impact power on these participants' motivations. Incredibly, there are four MFs belong to the Intrinsic Motivation category (i.e., MFs 15, 
$13,14,10)$, while the other three MFs belong to the Extrinsic Motivation category (i.e., MFs 5, 18, 19; details were presented in Table 2).

Second, apply the absolute criteria of correlation coefficients by Zhu (2012), the 15 motivation variables entered the EFA and MRA were examined. The results are: the Intrinsic Motivation structures showed low to moderately high positive correlation coefficients among the variables (i.e., $r$ ranged from .21 to .63). The extrinsic motivation structures showed low to moderately high positive correlation coefficients among the variables (i.e., $r$ ranged from .21 to .59). In addition, there are some motivation variables showed quite low correlation to one another (i.e., $r=.02-.11$ ). Hence, intrinsic motivation and extrinsic motivation possess a pretty similar impact power on these YBPs' participation motivations; however, in specific those intrinsic motivations, marginally higher impact power than those of extrinsic motivations. Up to this point, it can be concluded that: the ten intrinsic motivations in the AYBPPMQ (Zeng, 2019) were core motivation factors for these YBPs. Of cause, some extrinsic motivations could possess slightly higher impact powers than the other factors, and other extrinsic motivations could possess slightly lower impact power than the other factors too. Based on these findings, youth basketball coaches, trainers, or administrators should analyze their players' specific situation and implement the findings wisely and suitably.

Third, the follow up factorial MANOVA test discovered that: 10 out of 19 comparisons in the Goal-Setting (for professional, for non-professional) element showed significant differences, wherein 8 comparisons reached significance at $p<.05$ level and 2 comparisons reached significance at $p<.01$ level with for-professional scored significantly higher than for non-professional. For instance: MF5 For meet friends, MF7 Contest winners, MF11 Foster self-esteem and MF 12 Improve reputation. Meanwhile, the MF10 Becomes a professional player, and MF18 Becomes a coach reached a significant difference at $p<.01$ levels. It is understandable that when the players were facing those ten motivation factors, their reaction or responses will different, the players who set up their goal to become a professional will be deeper, even filled with passion, because they had stronger feelings about these motivation factors. They were actually motivated to engage in the basketball practices and competitions day by day, week by week, and they are in love with their training environment, teammates, and their teams demonstrate a cohesion atmosphere. Additionally, these YBPs are serious about their 'Athlete career credit' as the requirement for playing at the collegiate level: a player will need to accumulate his/her athlete career credit, "which will determine his/her chances of competing at the college level, and ultimately the professional level if his/her talented enough" (p. 2, NYCYBL, 2018).

All of these factors are what these YBPs need to accomplish to attain their basketball-star dream. This is why these ten MFs possessed significantly higher impact power on their participation motivations. To the MF10 Become a professional player, the MF12 To improve reputation, and the MF18 To become a basketball coach; again, the results were For-professionals scored significantly higher than those of For nonprofessional (also see Table 3). What would be the reasons behind those significant 
differences? We believe there should be some very special factors or reasons behind this. When a YBP sets up his/her goal to become a professional player, he/she will be more serious and focused and want to meet his/her teammates and make new teammates. During their practices and competitions, they will try their best to be on the top of his/her team because he/she wants to become a winner; this will help build up his/her biography and establish his/her prestige. Moreover, if they achieve their goal - become a professional player, they will very well satisfy their family's will. This is why they scored significantly higher than those who set-up their goal for playing as a non-professional.

On the other hand, to those YBPs who set up their goal For non-professional, when they facing those motivation factors (such as: meets friends, contest winners, get recognition, establish prestige, and become a professional player) their reactions were not as exciting as those want to become professional players; their excitement level was lower than those For-professional players; they might have to practice, receiving coaches' directions, facing challenges, and attending competitions differently. Another reason might be: usually, these types of basketball players have better academic performance and may only want to play at the university/college level (e.g., the NCAA Division II or III, 2018). Therefore, in the current stage, motivation factors (MFs) such as contest winners, establish prestige, and become a professional are not what they concern about; hence, the results of this survey were reasonable, logical, and correspond to those youth basketball players' reality.

Not surprisingly, when comparing the MFs between the two Goal-settings, some interesting and unique facts came out: 1) the athletes who set up their goal forprofessional were significantly more appreciative of the MF7 contest winners; MF10 become a professional player; MF12 improve my own reputation than those who set up their goal for non-professional. What was the key reason for all of these? We strongly believe that it is still the Intrinsic Motivation factors cause because that is why those youth players really want to be. Successful or not, they must try their best.

It should be indicated that there are certain similarities and differences existing between the present study and previous studies. For example, using the previous studies' findings for the sport of tennis, soccer, and volleyball: A review of literature in motivation in Tennis (Miguel \& Machar, 2007); Research studies in youth athletes' participation motivations in soccer (Zeng et al. 2019); and Research studies in youth athletes' participation motivations in volleyball (Zeng, 2018a). Miguel and Machar (2007) summarized that: first, enjoyment, having fun, and passion for the sport was rated as the top three important MFs for the success of youth tennis players. Second, improving performance, keeping fit, and socializing was rated as the basic reasons for keeping them involved in the sport. Third, feeling important and popular, and earning rewards were ranked as lower influence motivations. Last, School/club/team atmosphere and having a good relationship with the coach was also ranked as less/lower important factors on players' motivation.

Next, research findings from youth soccer (Zeng et al. 2019) revealed that: the top five factors were Technical Content \& Unique-Value, for fun, to make new friends, for 
my biography, and to establish prestige, and the other five factors possessed lower impact power on those youth soccer player's motivation: get the recognition, to improve health, for one unique skill, to reduce troubles, to be a professional. Third, in the youth volleyball players' motivations study, Zeng et al. (2019) indicated that: the top five factors were Technical Content \& Unique-Value; To develop extraordinary skills, For gettinghealthier, For enjoyment, and To improve physical fitness. The middle five factors were To make new friends, To contest winners, To reduce working pressure, To reduce pressure from academic learning, and To foster self-esteem, etc.

As discussed above, although this study and those previous studies were conducted in different sports and countries, these findings have many similarities. Specifically, from top to medium impact power motivation factors (MFs) for these youth athletes keeping engaged in these sports were similar. Meanwhile, when comparing those MFs, such as feeling important and popular, earning rewards, team atmosphere and good relationship with the coach from those previous studies with the MFs of Technical content and value, Unique skills, For fun, For biography, For establishing prestige, Become a professional player, For self-esteem, To contest winners, and Become a basketball coach, from this study; it is clear that there are numerous differences between those previous studies and the current study.

As to the element of Supports (by parents or by school) among the comparison from the $19 \mathrm{MFs}$, the results revealed that there are six MFs showed significant difference at $P<.05$ level (i.e., MF5 To meet my friends, MF10 To become a professional player, MF11 To foster self-esteem, and MF17 To develop a unique sport skill) with the YBPs' Supporting by parent scored remarkable higher than those of Supporting by schools. This finding might implicate that parents who have a sports background, believed their children have athletic talents and had a wish their children become sports stars in the near future could provide their child more supports.

Furthermore, the exploratory factor analysis (EFA) and the multiple regression analysis (MRA) techniques can be effectively used for predicting the three basic psychological needs; and the Gender, Goal-setting, Supporting, Years play Basketball four related elements. The findings of this study indicated that EFA and MRA are the efficient ways for enhancing the instruction effects and management level of the physical educators (including coaches, instructors, administers, managers, and staff), the consequence should enable them to create better and more effective training/education program in the youth basketball. More specifically, this study examined the overall degree of the YBPs' three psychological needs satisfaction at their participation motivations. The result of MRA about the satisfaction degrees on Competence needs, Relatedness needs, and Autonomy needs reaching 76.7\% (see Table 5). This finding extends the literature by providing live pieces of evidence about the psychological mechanism associated with the YBPs' participation motivations that have not been explored previously in youth sports research.

According to the Self-Determination Theory (Ryan \& Deci, 2000), this finding may provide a guideline for the YBPs (direct by their coaches/instructors), in how to use the 
motivation traits to participate in their practices and competitions continually. The motivation traits of this sample' YBPs were summarized in Figure 1.

Figure 1: Traits of three basic psychological needs of the YBPs $(\mathrm{N}=253)$

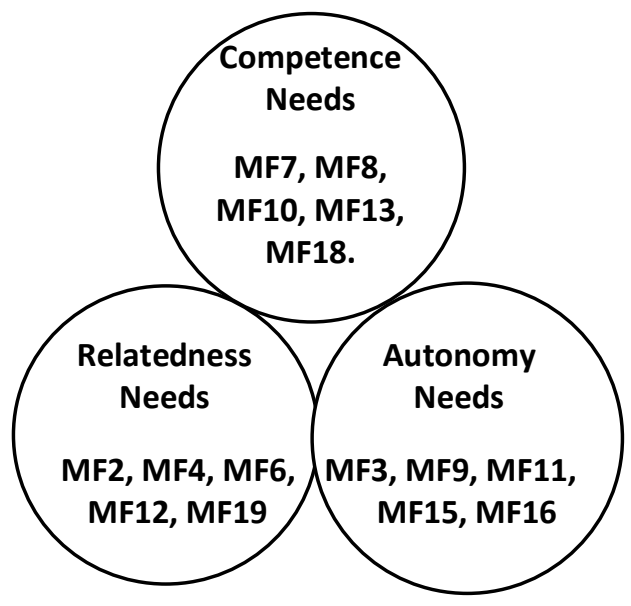

The keys for what the MFs represent:

MF2. 'for fun \& not boredom' MF3. 'for getting healthier body'

MF4. 'for enjoyment and happiness. MF6. 'to make new friends'

MF7. 'to contest winners' MF8. 'to shape body'

MF9. 'to improve health' MF10. 'for become a professional'

MF11. 'to foster self-esteem' MF12. 'to improve my reputation'

MF13. 'to establish prestige' MF15. 'to reduce pressure'

MF16. 'to reduce troubles' MF18. 'to become a coach.

MF19. 'to satisfy family will'

Note: a) The Competence Needs, Relatedness Needs, and Autonomy Need in the Self-Determination Theory (SDT) model were interpreted by Ryan and Deci (2000); and b) The 15 variables in this figure were selected/determined by the exploratory factor analysis.

In fact, the findings from the present study were consistent with the SelfDetermination Theory; that is, the participants in the current study were based on their own decision (or self-determined) to participate in their basketball practices and competitions. The overall effects of correlation coefficients between their three psychological needs and their participation motivations showed a positive relationship (see Table 3 and Table 4 also).

\section{Endnote and Recommendation}

This exploratory study provides new findings, grounded in the perspective of SelfDetermination Theory (Ryan \& Deci, 2000) predicted the relationships between the 19 motivation factors and the four related elements (Gender, Years play Basketball, Goalsetting, and Supports). Results specifically support the idea that three basic psychological needs contributed to the youth basketball players' participation-motivations, and these motivation factors can be used for predicting whether or not these youth basketball 
players (YBPs) will continually participate in their practices and competitions. The present study added a set of new data reflected the critical factors that truly motivated these YBPs participating in basketball practices and competitions. This study also identified what roles of the Gender, Years Play Basketball, Goal-setting, and Supports elements played, revealed that Gender and Years Play Basketball are not the determination elements but Supporting and Goal-setting elements are. Supporting and Goal-setting have a significant impact on the youth basketball players' participation motivations. By providing evidence on players who supported by their parents possess higher motivations than players who supported by their school; players who set-up their goal to be professionals possess higher motivations than those of set-up their goals to be non-professionals. This study added to the existing body of knowledge on the traits of the YBPs' participation motivations. These findings could be lively and meaningful examples for physical educators used in their sports pedagogy/sports management or physical education teacher education programs. When these works getting done, it will bring better or more perfective coaching, teaching, and sport management in the realm of youth basketball.

\section{Conclusion}

In conclusion, the elements of Gender and Years Play Basketball are not the determining factors but the Supports, and Goal-setting elements are; the YBPs who supported by their parents possess higher participant motivations than those of the YBPs who supported by their school/team. The YBPs who set up their goal for professionals possess higher participant-motivations than those players who set up their goal for non-professionals. The Intrinsic Motivations possess slightly higher impact power than those of the Extrinsic Motivations on these YBPs. The Exploratory Factor Analysis and the Multiple Regression Analysis techniques can be useful for helping educators (coaches/instructors) and administrators (program directors/managers) find out a more effective way to assist their players in maintaining or enhancing their participation-motivations, even reform or improve their basketball program. Youth basketball educators need to analyze their athletes' situations and employ these research findings correspondingly.

\section{Acknowledgements}

The following administrators and coaches provided remarkable help during the data collection of this study. Here, my deepest appreciation and greatest acknowledgment are giving to Dean Steven Riveras, Dean Andria Cuello, Coach Jose Garcia, and Coach Chris Gigliello; the same appreciation is also expressing to those coaches whose team were in this study, but they do not want their names to appear here. Without their enthusiasm supports, this study would not be possible! The author of this article: Howard Z. Zeng (D.P.E.). 


\section{Funding}

This research project was supported by Research Award \# Cycle 48 (Grant No. PSCCUNY 60702-00 48) of the City University of New York.

\section{Conflicts of Interest Statement}

The author declared no potential conflicts of interest with respect to the research, authorship, and/or publication of this article.

\section{About the Author}

Dr. Howard Z. Zeng is a Professor of Sport Pedagogy at Department of Kinesiology, Brooklyn College of The City University of New York, USA. His research interests are physical education pedagogy and sports science. He is a long time member of SHAPE AMERICA and AIESEP. By fare, he has published more than 60 articles in different academic journals.

\section{References}

Basketball Player Positions. Basketball Player Positions. Available: http://www.ducksters.com/sports/basketball/playerpositions.php 2017.

Breese HP, 1998. Participation motivation in ITF Taekwondo: A study of the central districts region [Master Theses]. Massey University, NZ.

Chen S, Sun H, Zhu X, \& Chen, A, 2014. Relationship between motivation and learning in physical education and after-school physical activity. Journal of Research Quarterly for Excise and Sport, 85: 468-477.

Child, D. 1990. The essentials of factor analysis, second edition. London: Cassel Educational Limited.

Claver F, ఏiménez R, Gil-Arias A, Moreno A, \& Moreno MP, 2017. The Cognitive and Motivation Intervention Program in Youth Female Volleyball Players. Journal of Human Kinetics. 59: 55-65.

Cox RH, 2011. Sport psychology: Concepts and application. Dubuque, Iowa: W.C. Brown.

Duricek M, Cynarski WL, 2017. Motivational incentives of all-round entertainers and types of psychical processes of participants during animation programs. Ido Movement for Culture. Journal of Martial Arts Anthropology, 17: 9-14, doi: 10.14589/ido.17.1.4

Faurschou B, 2015. History of Basketball - NBA hoops online. Available: https://nbahoopsonline.com/Articles/History1.html.

Geidne S, Quennerstedt M, Eriksson C, 2013. The youth sports club as a health-promoting setting: An integrative review of research. Scandinavian Journal of Public Health. 41: 269-283, doi: 10.1177/1403 494812473204. 
Gonzalez-Cutre D, Sicilia A, 2012. Motivation and Exercise Dependence: A Study Based on Self-Determination Theory. Journal of Research Quarterly for Excise and Sport, 83: 318-329.

Kaplan A, 2010. Intrinsic and Extrinsic Motivation. Available: http://www.education.com/reference/article/intrinsic-and-extrinsic-motivation/\#.

Lippitt E, 2012. Motivation, Need Support and Need Satisfaction in Youth Soccer Players. Master

Thesis. https://digitalcommons.Georgiasouthern.edu/cgi/viewcontent.cgi?article=2046\&c ontext=etd.

McPherson SC, 2015. Motivational climate and goal orientation in adolescent male football Players. Master Thesis. Clemson University.

Miguel, C., and Machar, M. R. (2007). Motivation in tennis. British Journal of Sport Medicine, 41: 769-772, doi: 10.1136/bjsm.2007.036285.

NBA Draft, 2016. 2003 NBA Draft where are they now? https://www.businessinsider.com/2003-nba-draft-where-are-they-now-2016-11.

National Collegiate Athletic Association (NCAA), 2018. College Basketball. https://en.wikipedia. org/wiki/ National Collegiate Athletic Association

New York City Department of Education (NYCDE), 2018). New York City School Portals. http://schools.nyc.gov/SchoolPortals/26/Q430/Academics/Athletics/default.htm.

New York City Youth Basketball League (NYCYBL), 2018. The New York City youth basketball league. http://www.nycyfl.com/home.

Official Report, 2010. The official report: Singapore 2010 Youth Olympic Games. https://www.olympic.org/singapore-2010.

Pintrich PR, Schunk D, 2002. Motivation in education: Theory, research and applications (Second ed.). Upper Saddle River, NJ: Prentice-Hall.

Ryan RM, Deci EL, 2000. Self-determination theory and the facilitation of intrinsic motivation, social development, and well being. American Psychologist, 55: 68-78. http://psycnet.apa.org/doi/10.1037/0003-066X.55.1.68.

Ryan RM, Frederick CM, Lepes D, Rubio D, Sheldon KS, 1997. Intrinsic motivation and exercise adherence. International Journal of Sports Psychology, 28: 355-354.

Smith AL, Balagurer I, Duda JL, 2006. Goal orientation profile differences on perceived motivational climate, perceived peer relationships, and motivation-related responses of youth athletes. Journal of Sports Sciences, 24: 1315-1327.

Stellion MB, Sinclair CD, 2013. Psychological predictors of children's recess physical activity motivation and behavior. Research Quarterly for Excise and Sport, 84: 167176.

Zeng HZ, 2018. Exploring of Youth Volleyball Players' Engagement Motivations and Health Related Behaviors. European Journal of Physical Education and Sport. 6: 80-94, doi: 10.13187/ejpe.2018.2.80

Zeng HZ, 2018. An Investigation of Youth Football Players' Participation Motivations and Health Related Behaviors. The Sport Journal, October 18. America's Sport University®. http://www.ussa.edu. 
Zeng ZH, 2019. A Study of Youth Martial Arts Athletes' Engagement Motivations and Their Health Related Behaviors. Ido Movement for Culture. Journal of Martial Arts Anthropology, 19: 20-33, doi: 10.14589/ido.19.1.2.

Zeng ZH, Meng WY, Sun P, Xie LS, 2019. A Study of Youth Soccer Players' Participation Motivation and Health Related Elements. The Physical Educator, 76: 327-355.

Zhu W, 2012. Sadly, the earth is still round ( $p<0.05)$. Journal of Sport and Health Science, 1: 9-11. 

be applied to their work. Under the terms of this license, no permission is required from the author(s) or publisher for members of the community to copy, distribute, transmit or adapt the article content, providing a proper, prominent and unambiguous attribution to the authors in a manner that makes clear that the materials are being reused under permission of a Creative Commons License. Views, opinions and conclusions expressed in this research article are views, opinions and conclusions of the author(s). Open Access Publishing Group and European Journal of Physical Education and Sport Science shall not be responsible or answerable for any loss, damage or liability caused in relation to/arising out of conflict of interests, copyright violations and inappropriate or inaccurate use of any kind content related or integrated on the research work. All the published works are meeting the Open Access Publishing requirements and can be freely accessed, shared, modified, distributed and used in educational, commercial and non-commercial purposes under a Creative Commons attribution 4.0 International License (CC BY 4.0). 